\title{
Distributed Control Scheme to Regulate Power Flow and Minimize Interactions in Multiple Microgrids
}

\author{
M. J. Hossain, Senior Member, IEEE, M. A. Mahmud, Member, IEEE, H. R. Pota, Member, IEEE, \\ N. Mithulananthan, Senior Member, IEEE and R. C. Bansal, Senior Member, IEEE
}

\begin{abstract}
In this paper, a distributed controller is designed for regulating power flow among multiple microgrids in islanded modes with variable renewable energy sources (RESs) while minimizing dynamic interactions. The controller is designed in such a way that it has the capability of gathering information from the neighboring microgrids. This paper also analyzes the dynamic interactions among multiple interconnected microgrids during an islanded mode of operation based on the detailed dynamical model of microgrids. Both modal analysis and nonlinear simulations are used to identify the critical issues that might degrade the stability under different operating conditions. From the simulation results, it is found that multiple microgrids operated with local controllers can significantly affect the stability and damping performance. The proposed control scheme is implemented on a test distribution system with multiple microgrids and time-domain simulations are performed to verify its effectiveness of the control scheme. It is found that the designed controller provides excellent performance in minimizing the negative interactions through the regulation of power flow among multiple microgrids in the islanded mode.
\end{abstract}

Keywords-Multiple microgrids, distributed control, dynamic interactions, islanding operation, power flow regulation.

\section{INTRODUCTION}

$\mathbf{C}$ Ontrol of islanded microgrids with non/less inertial renewable energy sources (RESs) is a great challenge for the future power industry. Without a decent control mechanism, serious problems, for example, voltage profile degradation, frequency instability and loss of synchronization can occur in the system. Clustered and agent-based control are proposed in the literature for controlling multiple microgrids [1][3]. A cooperative control approach is proposed for controlling voltage by sharing reactive powers among groups of distributed generator (DG) units in [4]. The authors in [5] suggest an optimal reactive power control strategy. However, it has a centralized architecture which requires expensive and reliable communication systems. Recently, a decentralized voltage

M. J. Hossain is with the Griffith School of Engineering, Griffith University, Gold Coast, QLD-4215, Australia. email: j.hossain@griffith.edu.au.

M. A. Mahmud is with the Faculty of Engineering and Industrial Sciences, Swinburne University of Technology, Hawthorn, VIC 3122. Email: mmahmud@swin.edu.au

H. R. Pota is with the School of Engineering and Information Technology, The University of New South Wales, Canberra, ACT-2600, Australia. email: h.pota@adfa.edu.au.

N. Mithulananthan is with the School of ITEE, The University of Queensland, St. Lucia, Brisbane, QLD-4072, Australia. e-mail: mithulan@itee.uq.edu.au.

R. Bansal is with the Dept. of EECE, University of Pretoria, South Africa. Email: rcbansal@ieee.org control is proposed to minimize distribution power losses in microgrids [6]-[8]. PI controllers are used in [6]-[8] to design voltage and power controllers which work well for a particular system operating conditions. During large disturbances, for example, islanding from grid-connected mode, this type of controllers cannot ensure stable operation as these are not designed by considering the information from neighboring microgrids.

Distributed control is getting popular for controlling clustered microgrids with RESs and energy storage systems (ESSs) as it is significantly difficult to manage and control a large number of distributed generator (DG) units using either centralized or decentralized control. It uses local communication networks and includes the positive features of both centralized or decentralized control while limiting their drawbacks [9]. A self-organizing distributed control for the PV unit is proposed in [10] for power flow control in a distribution system without considering the dynamics of inverters and PV units. The authors in [11] propose a distributed control for power sharing and voltage control in DC microgrids. A model predictive distributed control is proposed in [12] in order to maintain the voltage profile of a multi-area power system within the acceptable bounds. The system nonlinearity and dynamics are completely neglected in [11], [12]. A distributed hierarchical control architecture for automatic generation control is proposed in [13] for large-scale power systems by considering the dynamics of synchronous generators but this is not the case for microgrids in the islanded mode of operations.

When microgrids operate in an islanded mode, the main sources of supplying power to loads are variable RESs rather than synchronous generators. During the grid-connected operation of microgrids, most of the active and reactive power support are provided by the synchronous generator and the excess power from RESs is stored into energy storage systems (ESSs). But during the islanded operation, the amount of active power may be adequate due to the power supply from ESSs and RESs and in this situation, the requirement of reactive power may not be fulfilled. In this case, the contribution of var compensators such as static compensators (STATCOMs) are essential.

This paper investigates the dynamic interactions among different interconnected microgrids. Each microgrid subsystem is integrated with RESs and a STATCOM/ESS and these microgrids negatively interact with each other due to the lack of appropriate power regulation. The amount of exact power regulation can easily be obtained by regulating the active power of ESSs and reactive power of STATCOMs which are 


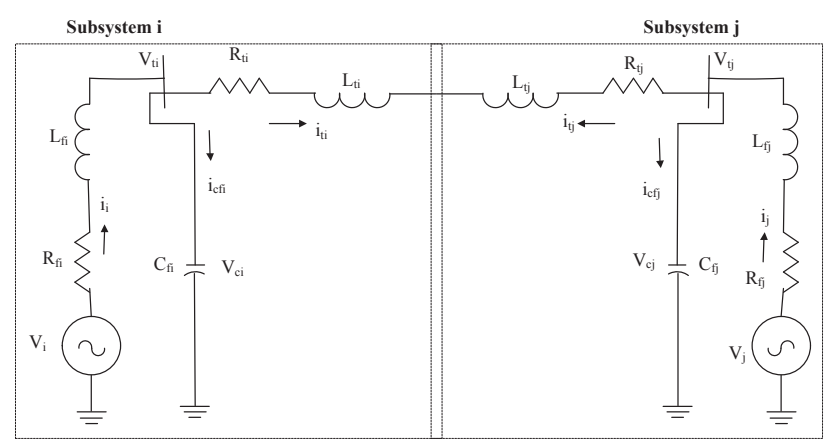

Fig. 1. Single line diagram of microgrid subsystems.

connected to each microgrid subsystem. The main target of this paper is to develop a distributed control scheme to achieve the desired power regulation by controlling STATCOM/ESS of each microgrid which minimizes negative interactions.

\section{Modeling OF MiCROGRIDS}

An accurate nonlinear dynamical model of a microgrid is significantly important for stability analysis and controller design. The model of a microgrid can have different level of complexities depending upon modeling goals. In this paper full order models of fast acting power electronics converters are considered. Each DG unit is represented by a DC voltage source, a voltage source converter (VSC) and a filter. Fig. 1 shows the single line diagram of two microgrid subsystems connected at $i^{\text {th }}$ and $j^{\text {th }}$ nodes in an interconnected microgrids. Since DG units are considered as voltage source, $V_{d c}$ is an ideal DC voltage source equivalent to a DG. In Fig. $1, R_{f}$ is the equivalent resistance accounting for the switching and transformer losses, $L_{f}$ is the filter and leakage reactance of the transformer, $C_{f}$ is the filter capacitance, $V_{c}$ is the voltage across the filter capacitor, $R_{t}$ and $L_{t}$ are line resistance and inductance, respectively.

The mathematical modeling of $i$ th converter in $d q$ frame is given by [14]:

$$
\begin{aligned}
& L_{\mathrm{f}_{i}} \dot{I}_{\mathrm{d}_{i}}=V_{\mathrm{d}_{i}}-R_{\mathrm{f}_{i}} I_{\mathrm{q}_{i}}+\omega_{i} I_{\mathrm{q}_{i}} L_{\mathrm{f}_{i}}-V_{\mathrm{td}_{i}} \\
& L_{\mathrm{f}_{i}} \dot{I}_{\mathrm{q}_{i}}=V_{\mathrm{q}_{i}}-R_{\mathrm{f}_{i}} I_{\mathrm{d}_{i}}-\omega_{i} I_{\mathrm{d}_{i}} L_{\mathrm{f}_{i}}-V_{\mathrm{tq}_{i}} \\
& L_{\mathrm{t}_{i}} \dot{I}_{\mathrm{td}_{i}}=V_{\mathrm{td}_{i}}-R_{\mathrm{t}_{i}} I_{\mathrm{tq}_{i}}+\omega_{i} I_{\mathrm{tq}_{i}} L_{\mathrm{t}_{i}}-V_{\mathrm{td}_{j}} \\
& L_{\mathrm{t}_{i}} I_{\mathrm{tq}_{i}}=V_{\mathrm{tq}_{i}}-R_{\mathrm{t}_{i}} I_{\mathrm{td}_{i}}-\omega_{i} I_{\mathrm{td}_{i}} L_{\mathrm{t}_{i}}-V_{\mathrm{tq}_{j}} \\
& C_{i} V_{\mathrm{td}_{i}}=I_{\mathrm{d}_{i}}-I_{\mathrm{td}_{i}}+\omega_{i} V_{\mathrm{tq}_{i}} C_{i}-I_{\mathrm{cd}_{i}} \\
& C_{i} V_{\mathrm{tq}_{i}}=I_{\mathrm{q}_{i}}-I_{\mathrm{tq}_{i}}-\omega_{i} V_{\mathrm{td}_{i}} C_{i}-I_{\mathrm{cq}_{i}}
\end{aligned}
$$

where $\left.V_{i}=\sqrt{(} V_{\mathrm{d}_{i}}^{2}+V_{\mathrm{q}_{i}}^{2}\right)$. The real and reactive power power injected by the DG units at the $i$ th bus is given as:

$$
\begin{aligned}
P_{i} & =\frac{3}{2}\left(V_{\mathrm{tq}_{i}} I_{\mathrm{tq}_{i}}+V_{\mathrm{td}_{i}} I_{\mathrm{td}_{i}}\right) \\
Q_{i} & =\frac{3}{2}\left(V_{\mathrm{tq}_{i}} I_{\mathrm{td}_{i}}-V_{\mathrm{td}_{i}} I_{\mathrm{tq}_{i}}\right)
\end{aligned}
$$

Fast and independent active and reactive power support provided by a STATCOM/energy storage system (ESS) can significantly enhance the performance of microgrids. The main

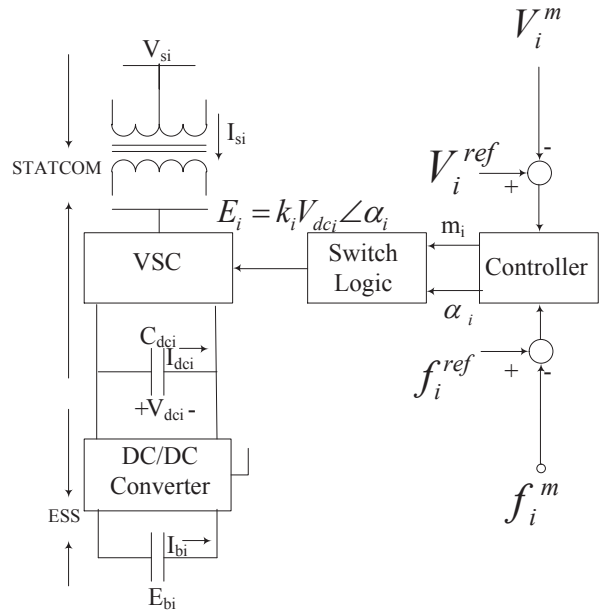

Fig. 2. STATCOM/ESS control strategy.

components of the STATCOM/ESS shown in Fig. 2 are a normal STATCOM and an ESS. A normal STATCOM is comprised of a coupling transformer, a VSC, and a DClink capacitor (usually an electrolytic). The DC-link capacitor provides voltage supports for the VSC and the DC chopper. The ESS is comprised of a battery and a bi-directional DC-DC buck-boost converter to control the charging and discharging of the battery. The aim of this module is to store energy in the battery and then deliver that energy to the grid via the DClink when required. The DC-DC converter operates in buck mode to recharge the battery, whereas boost mode transfers the stored energy to the DC-link.

The mathematical modeling of VSC of the STATCOM is given as [15]:

$$
\begin{aligned}
L_{\mathrm{s}_{i}} \dot{I}_{\mathrm{sd}_{i}} & =-R_{\mathrm{s}_{i}} I_{\mathrm{sd}_{i}}+I_{\mathrm{sq}_{i}} \omega_{i}+V_{\mathrm{sd}_{i}}-V_{\mathrm{dc}_{i}} s_{\mathrm{d}_{i}} \\
L_{\mathrm{s}_{i}} \dot{I}_{\mathrm{sq}_{i}} & =-R_{\mathrm{s}_{i}} I_{\mathrm{sq}_{i}}+I_{\mathrm{sd}_{i}} \omega_{i}+V_{\mathrm{sq}_{i}}-V_{\mathrm{dc}_{i}} s_{\mathrm{q}_{i}} \\
\dot{v}_{\mathrm{dc}_{i}} & =-\frac{P_{\mathrm{s}_{i}}}{C_{i} v_{\mathrm{dc}_{i}}}-\frac{v_{\mathrm{dc}_{i}}}{R_{\mathrm{c}_{i}} C_{i}},
\end{aligned}
$$

where $v_{d c_{i}}$ is the capacitor voltage, $C_{\mathrm{dc}_{i}}$ is the DC capacitor, $R_{\mathrm{c}_{i}}$ is the internal resistance of the capacitor, and $P_{\mathrm{s}_{i}}$ is the power supplied by the system to the STATCOM to charge the capacitor. The STATCOM terminal AC voltage is $E_{i}=k_{i} v_{d c_{i}} \angle \alpha_{i}$ where $\alpha_{i}$ is the bus angle of the STATCOM in the reduced network, and $k_{i}=\sqrt{\frac{3}{8}} m_{i}$, where $m_{i}$ is the modulation index.

\section{Microgrid STRUCTURE AND CASE STUdY}

The smart operation of a microgrid can be achieved using fast control of hundreds of small individual DG unit. However, it requires real-time information of each DG unit and critical loads. This complexity can be greatly reduced by interconnecting multiple microgrids. A STATCOM with an ESS is connected with a microgrid to control both the active and reactive power. Inverters with ESSs could be used to supply power among interconnected microgrids and tie-line power 


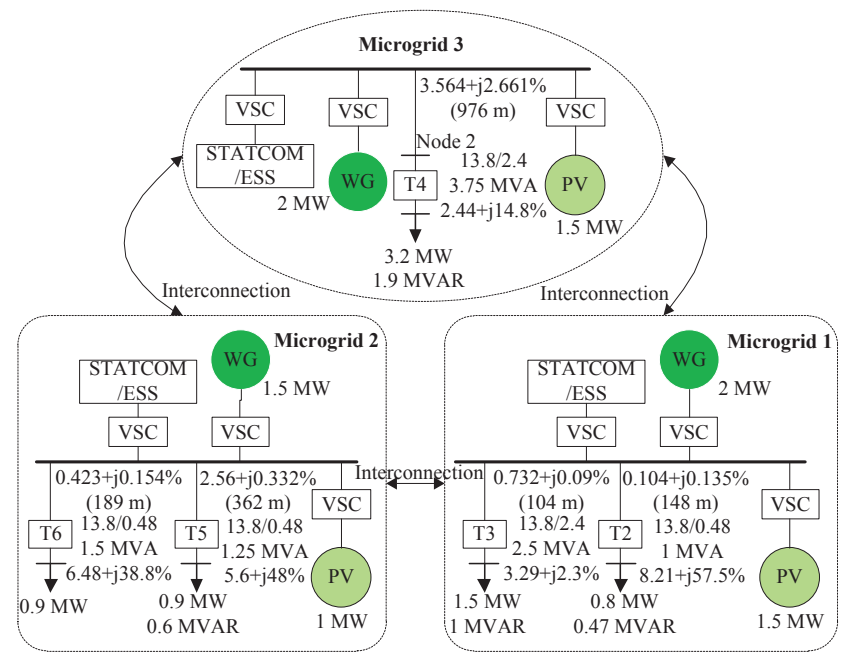

Fig. 3. Single line diagram of the test system.

could be controlled by these inverters which are done in this paper. The power transfer among the multiple microgrids can be controlled depending on the magnitude of frequency.

The test system considered in this paper, as shown in Fig. 3, consists of three interconnected microgrids. Each microgrid consists of a PV unit, a fully controlled wind generator (WG), a STATCOM/ESS and mixture of static and dynamic loads. The capacity of the STATCOM is $20 \mathrm{kVA}$ and ESS is 800 Ah. Under normal operating conditions $0.8 \mathrm{MW}$ power is delivered from microgrid 1 to microgrid $3,0.4 \mathrm{MW}$ from microgrid 2 and 3 and no power between microgrids 2 and 3 . A case study is conducted with the conventional controllers which are designed locally without sharing any information with each other [16]. A three-phase fault is applied at the point of common coupling (PCC) in microgrid 3 at $1 \mathrm{~s}$ and subsequently cleared after $100 \mathrm{~ms}$. Figure 4 shows the power flow from microgrid 1 to microgrid 3 when only two microgrids ( 1 and 3 ) and three microgrids are in operation. It is evident from Fig. 4 that the power flow is oscillatory and take longer time to return to the steady-state condition. The addition of third microgrid reduces the system damping and has negative impacts on the dynamic performance of the system. In order to get the insight of the problem a small-signal analysis is carried out from where it is found that states related to all STATCOM/ESS controllers contribute significantly in the dominating mode.

In a microgrid with thousands of DG units, for example, PV units, wind generators, ESSs and flexible AC transmission system (FACTS) devices, the charging and discharging of the batteries need to be adjusted as the output powers of wind and PV generators are variable due to fluctuations in wind speed and sunlight. It will be difficult to determine and maintain a feasible operating conditions if all the DG units operate independently with decentralized controllers. The centralized control scheme is also not practical and reliable as it requires global collection and exchange of information. Distributed controllers are capable of collecting and exchanging informa-

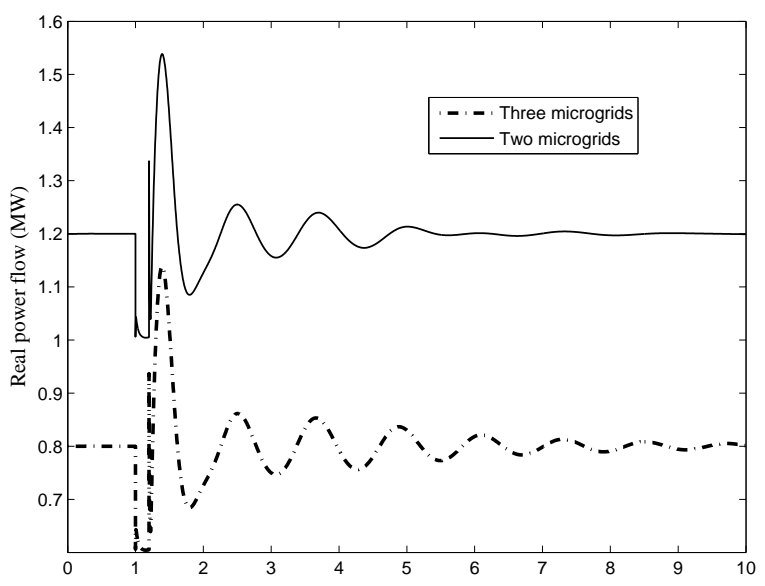

Fig. 4. Power flow from microgrid 1 to microgrid 3.

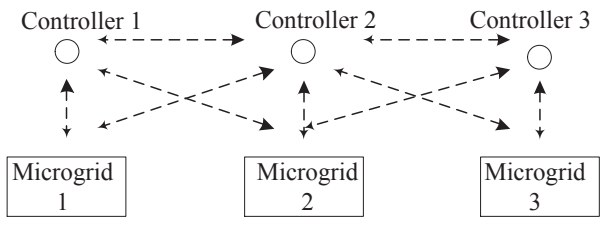

Fig. 5. Distributed controller.

tion among different microgrid subsystems and the design of a distributed controller is shown in the following section.

\section{Control Strategy}

A distributed control approach as shown in Fig. 5 is proposed to handle the numerous distributed energy resource (DER) units. The information among neighboring subsystems can be exchanged using the local communication network which requires less efforts as compared to the centralized control.

The DER units can be represented as follows by linearizing equations (1-3):

$$
\begin{aligned}
\dot{x}_{i} & =A_{i} x_{i}+B_{u_{i}} u_{i}+B_{\omega_{i}} \omega_{i} \\
z_{i} & =C_{z_{i}} x_{i}+D_{z u_{i}} u_{i} \\
y_{i} & =C_{y_{i}} x_{i}+D_{y \omega_{i}} \omega_{i}
\end{aligned}
$$

where $x_{i}=\left[I_{d_{i}}, I_{q_{i}}, I_{t d_{i}}, I_{t q_{i}}, V_{t d_{i}}, V_{t q_{i}}, I_{d s_{i}}, I_{q s_{i}}, V_{d c_{i}}\right]$ is the state vector, $u_{i}=\left[k_{i}, \alpha_{i}\right]$ is the control input and $\omega_{i}$ is the disturbance input and $z_{i}$ is the performance output of the $i$ th agent. The matrices $A_{i}, B_{u_{i}}, C_{z_{i}}$ and $C_{y_{i}}$ are constant and known, expressing the nominal subsystem model. The matrices $B_{\omega_{i}}, D_{z u_{i}}$ and $D_{y \omega_{i}}$ represent unmodeled part of the nonlinear terms, disturbance imposed by the system models, and disturbances due to unmodeled parts. The matrix $A_{i}$ is calculated at nominal operating points and the change in solar radiation and wind is modeled as a disturbance input to the system.

The STATCOM/ESS controller is depicted in Fig. 2 which provides real power and frequency command to achieve the 
desired system response during the transient period. The controller converts the power and frequency commands into pulsewidth modulation (PWM) switching signals for the STATCOM/ESS. The designed controller regulates the modulation gain $m_{i}$ and firing angle $\left(\alpha_{i}\right)$. The firing angle $\alpha_{i}$ mainly affects the variation of the active power exchanged among different microgrid clusters. Therefore, the function of the active power control is to meet the active power demand of the system during transients. The other output of the controller is the duty cycle ratio $m_{i}$ which mainly regulates the magnitude of the STATCOM's output voltage and therefore the system voltage. The reference frequency can be calculated from the state of charge (SOC) of the battery. The active power flow among the microgrids are controlled by the voltage phase angle through the regulation of firing angle $\left(\alpha_{i}\right)$ as shown in Fig. 2. Frequency is used as the distinction index to maintain power flow among microgrids both in transient and steadystate conditions.

The proposed controls for the STATCOM/ESS and the inverter are given as [17]:

$$
\dot{x}_{c}=A_{c} x_{c}+B_{c} y(t), u=C_{c} x_{c}+D_{c} y(t),
$$

where

$$
\begin{aligned}
A_{c_{i}} & =H_{21}^{-T}\left(V_{i}-\left[\begin{array}{ll}
H_{11}^{T} & R_{i}
\end{array}\right]\left[\begin{array}{cc}
A_{i} & B_{u_{i}} \\
C_{y_{i}} & 0
\end{array}\right]\left[\begin{array}{c}
G_{11} \\
S_{i}
\end{array}\right]\right) G_{21}^{-1} \\
B_{c_{i}} & =H_{21}^{-T} R_{i}, C_{c_{i}}=S_{i} G_{21}^{-1}, D_{c_{i}}=0 .
\end{aligned}
$$

where $G_{11}, H_{11}, R_{i}, S_{i}$ and $V_{i}$ are the optimization variables which are determined using the linear matrix inequality (LMI) as described in [17]. It is assumed that each microgrid can only communicate with its nearest neighbors and the Laplacian matrix is chosen as:

$$
\mathcal{A}=\left[\begin{array}{ccc}
2 & -1 & -1 \\
-1 & 2 & -1 \\
-1 & -1 & 2
\end{array}\right]
$$

The objective is to design a distributed controller given in equations (5) and (6) such that the closed system is quadratically stable and achieves a prescribed level of disturbance attenuation in the $H_{2}$ norm, i.e., $\left\|T_{w z}\right\|_{H_{2}}<\gamma$. In this paper $\gamma=0.78$.

\section{CONTROL DESIGN ALGORITHM AND PERFORMANCE EVALUATION}

The design steps of the output-feedback distributed controllers for the nominal system are given below:

- Model the system using equations (1)-(3) and solve it for the steady-state.

- Determine the best input/output variables for the controller from the residues which are the product of system controllability and observability Gramians.

- Linearize the system with the best input and output signals and determine nominal system matrices given in equation (4).

- Reformulate the nonlinear system using the technique proposed in Section IV and determine the other matrices given in equation (4).

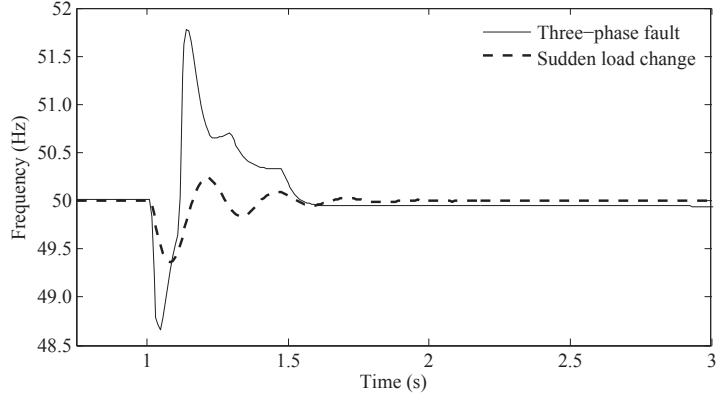

Fig. 6. Frequency response in microgrid 3.

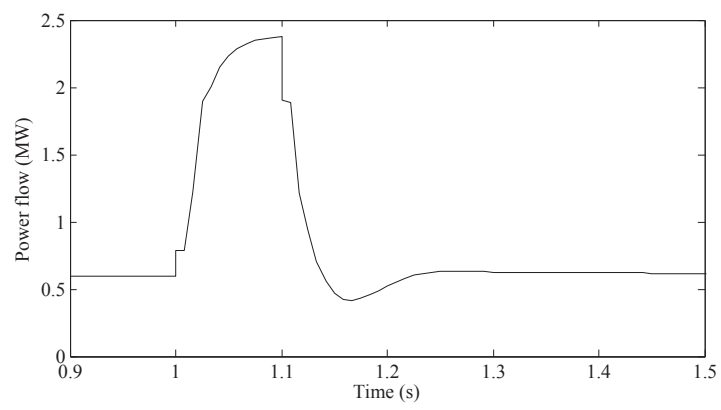

Fig. 7. Power flow from microgrid 1 to microgrid 3.

- Solve the linear matrix equality given in [17] to obtain $G_{11}, H_{11}, R_{i}, S_{i}$ and $V_{i}$ in equation (6).

- The controller is given by equations (5) and (6).

The objective of this section is to investigate the performance of the designed controller under various operating conditions on the test system as shown in Fig. 3. The designed controller is implemented by using PSCAD by considering two case studies: (i) severe three-phase fault in one of the microgrids and (i) sudden changes in load demand in a microgrid.

\section{A. Severe three-phase fault}

A severe three-phase fault is applied at $1 \mathrm{~s}$ at the interconnection between microgrids 1 and 3. Fig. 6 shows the frequency response in microgrid 3 (solid line). In this case, the active power flow between microgrid 1 and 3 is shown in Fig. 7. It is clear from the figures that the proposed controller ensures stable operation during severe transients and the postfault condition. Each STATCOM/ESS controller autonomously and effectively responds to the changes in system configurations and stabilizes it within a second without any steady-state error.

\section{B. Superiority to unknown load dynamics}

In this case study, an extra load with unknown dynamics is connected in microgrid 3 . As the voltage will reduce due to the increase in the load the controller will act quickly to increase the reactive power supply. The STATCOM/ESS will provide extra active power and interconnection inverter will supply the 


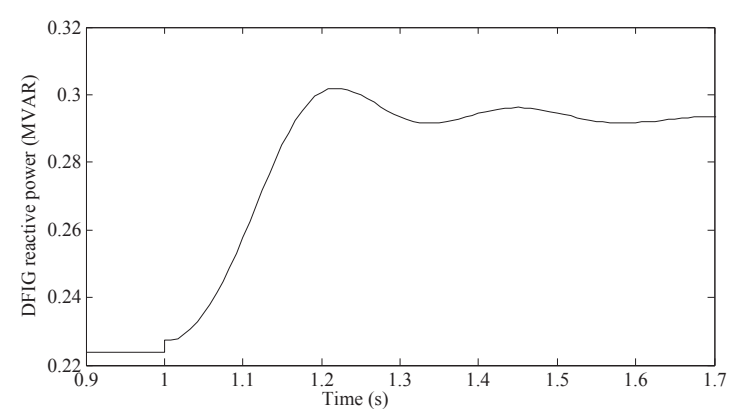

Fig. 8. Reactive power output of STATCOM/ESS in microgrid 1.

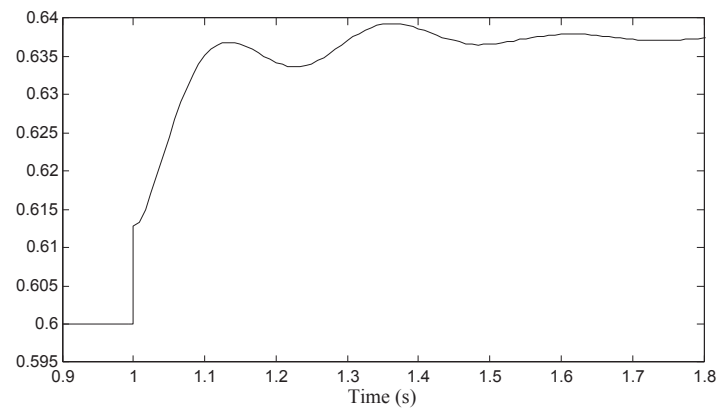

Fig. 9. Power flow from microgrid 2 to microgrid 3.

extra reactive power to balance the load. Fig. 8 shows the reactive power output of the STATCOM/ESS in microgrid 1. From Fig. 8, it is clear that other microgrids cooperate and share the power flow effectively to stabilize the voltage due the increases in loads in microgrid 1 . The frequency response in microgrid 3 due to the changes in loads is also shown in Fig. 6 (dotted line) and Fig. 9 shows the power flow from microgrid 2 to microgrid 3. From Fig 9 it is found that the designed controller provides excellent performance in terms of damping and settling time.

\section{CONCLUSIONS}

In this paper a systematic procedure for designing distributed controllers is presented in order to regulate power flow among multiple islanded microgrids. The proposed controller utilizes a distributed $\mathrm{H}_{2}$ control strategy and the appropriate inclusion of disturbances in the design process enhances the performance of the controller by minimizing dynamic interactions. The designed controller guarantees stability in the post-fault and the power balance of the system under extreme operating conditions. It provides feasible and smooth operation of coupled microgrids and load sharing among them. The simulation studies validate the desired performance of the microgrid subject to severe three-phase fault and sudden load variations which proves the efficacy and superiority of the designed controller. The future aim of this work is to design the controller considering the dynamics of nonlinear and voltage sensitive loads.

\section{REFERENCES}

[1] A. L. Dimeas and N. D. Hatziargyriou, "Agent based Control for Microgrids," IEEE PES General Meeting, 24-28 Jun. 2007.

[2] C. M. Colson and M. H. Nehrir, "Comprehensive Real-Time Microgrid Power Management and Control With Distributed Agents," IEEE Trans. on Smart Grid, vol. 4, no. 1, pp.617-627, Mar. 2013.

[3] A. Bidram, A. Davoudi, F. L. Lewis and J. M. Guerrero, "Distributed Cooperative Secondary Control of Microgrids Using Feedback Linearization," IEEE Trans. on Power Systems, vol. 28, no. 3, pp. 34623470, Aug. 2013.

[4] A. Maknouninejad, Z. Qu, J. Enslin and N. Kutkut, "Clustering and cooperative control of distributed generators for maintaining microgrid unified voltage profile and complex power control," IEEE PES Transmission and Distribution Conference and Exposition, 7-10 May 2012.

[5] A. G. Tsikalakis and N. D. Hatziargyriou, "Centralized Control for Optimizing Microgrids Operation," IEEE Trans. on Energy Conversion, vol. 23, no. 1, pp. 241-248, Mar. 2008.

[6] H. Liang, B. J. Choi, W. Zhuang and X. Shen, "Stability enhancement of decentralized inverter control through wireless communications in microgrids," IEEE Trans. on Smart Grid, vol. 4, no. 1, pp. 321-331, Mar. 2013.

[7] P. H. Divshali, A. Alimardani, S. H. Hosseinian and M. Abedi, "Decentralized cooperative control strategy of microsources for stabilizing autonomous VSC-based microgrids," IEEE Trans. on Power Systems, vol. 27, no. 4, pp. 1949-1959, Nov. 2012.

[8] C. Ahn and H. Peng, "Decentralized voltage control to minimize distribution power loss of microgrids," IEEE Trans. on Smart Grid, vol. 4, no. 3, pp. 1297-1304, Sept. 2013.

[9] P. Massioni and M. Verhaegen, "Distributed control for identical dynamically coupled systems: A decomposition approach, IEEE Trans. on Automatic Control, vol. 54, no. 1, pp. 124-135, Jan. 2009.

[10] H. Xin, Z. Qu, J. Seuss, and A. Maknouninejad, "A self-organizing strategy for power flow control of photovoltaic generators in a distribution network," IEEE Trans. on Power Systems, vol. 26, no. 3, pp. 1462-1473, Aug. 2011.

[11] S. Anand, B. G. Fernandes and M. Guerrero, "Distributed control to ensure proportional load sharing and improve voltage regulation in lowvoltage DC microgrids," IEEE Trans. on Power Electronics, vol. 28, no. 4, pp. 1900-1913, Apr. 2013.

[12] M. Moradzadeh, R. Boel and L. Vandevelde, "Voltage coordination in multi-area power systems via distributed model predictive control," IEEE Transa. on Power Systems, vol. 28, no. 1, pp. 513-521, Feb. 2013.

[13] L. D. Marinovici, J. Lian, K. Kalsi, P. Du and M. Elizondo, "Distributed hierarchical control architecture for transient dynamics improvement in power systems," IEEE Trans. on Power Systems, vol. 28, no. 3, pp. 3065-3074, Aug. 2013.

[14] Y. A.-R. I. Mohammad and A. A. Radwan, "Hierarchical control system for robust microgrid operation and seamless mode transfer in active distribution systems, IEEE Trans. on Smart Grid, vol. 2, no. 2, pp. 352-362, Jun. 2011.

[15] M. J. Hossain, H. R. Pota, V. Ugrinovskii and R. A. Ramos, "Simultaneous STATCOM and pitch angle control for improved fault ride-through capability of fixed-speed wind turbines," IEEE Trans. on Sustainable Energy, vol. 1, no. 3, pp. 142-151, Oct. 2010.

[16] Y. Hida, K. Koyanagi, R. Yokoyam, S. Nagata, K. Nakao and T. Hirai, "Electricity cluster-oriented network: a grid-independent and autonomous aggregation of microgrids," Proc. of the International Symposium on Modern Electric Power Systems, 20-22 Sep. 2010.

[17] R. Ghadami and B. Shafai, "Distributed $\mathrm{H}_{2}$ control of multi-agent dynamic systems: Continuous-time case," American Control Conference, Jun. 30-Jul. 02, 2010. 